\title{
análisis híbrido
}

HEINZ HOSSDORF, ingeniero civil

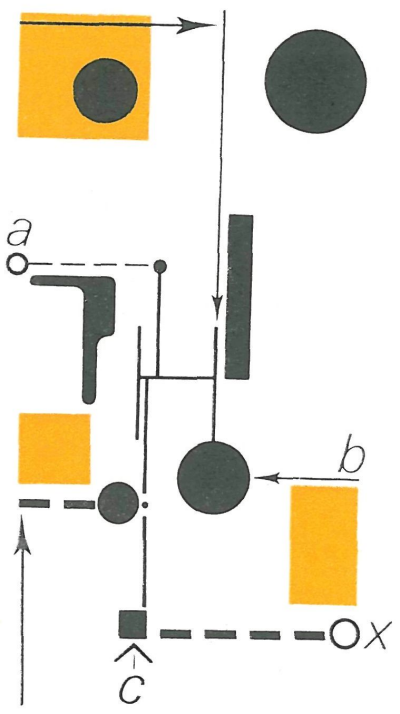

sinopsis En los últimos años se ha desarrollado, paralelamente al Método de los Elementos Finitos, otro especiaimente
concebido para el cálculo de estructuras elásticas complejas: el Análisis Híbrido. Este nuevo y potente método es todavía poco conocido, incluso entre especialistas, aunque desde hace algún tiempo se aplica ya con éxito

EI Análisis Hibrido es, en muchos casos, preferible a los métodos numéricos puros, tanto por razones económicas como por razones técnicas, entre las que destaca su más amplio campo de aplicación.

Aunque su planteamiento es conceptualmente simple, el método requiere una tecnología muy especializada y un sistema de programas de cálculo electrónico que no podrían ser descritos, en detalle, en un artículo tan corto. sus posibilidades mostrando su aplicación a algunos casos concretos. Para un tratamiento más detallado del Análisis Hibrido y de las correspondientes técnicas de ensayo, puede

\section{¿QUE ES EL ANALISIS HIBRIDO?}

El ensayo en modelo reducido tradicional presenta, frente al cálculo con ordenador electrónico, los inconvenientes de su premiosidad y elevado precio. Ello es debido a la necesidad de materializar físicamente, esto es, mediante "hardware", cada uno de los elementos constitutivos del problema estructural, creando mecanismos que simulen su comportamiento. Así, para representar la topología de la estructura y las características mecánicas del material hay que preparar en laboratorio un modelo a escala. Para representar las condiciones de borde o sustentación de la estructura, se recurre a dispositivos especiales: en el caso de apoyos elásticos, por ejemplo, a muelles con las constantes elásticas que correspondan. Igualmente deben representarse las distintas hipótesis de carga que actúan sobre la estructura, cargando mecánicamente el modelo con fuerzas repartidas sobre el mis- mo de acuerdo con las leyes prefijadas en cada caso.

Con el empleo del ordenador desaparecen estas dificultades. Todo se resuelve mediante la programación, es decir, el "software". La formulación del problema, desde la descripción de la estructura y sus condiciones de borde hasta la definición de las hipótesis de carga, es puramente numérica. El cálculo se realiza siguiendo programas generales previamente establecidos, que suministran los resultados deseados.

Hoy día, por tanto, se tiende a emplear el modelo reducido sólo en aquellos casos excepcionales en los que los métodos numéricos no son capaces de representar satisfactoriamente el comportamiento elástico de la estructura, a causa de la complejidad de la misma. 
El Análisis Híbrido, como su propio nombre indica, aparece como una simbiosis entre el ensayo en modelo tradicional y el cálculo numérico en ordenador (véase la tabla 1), que pretende combinar, de forma óptima, las ven tajas de ambos sistemas. Con ello se hace posible el aprovechamiento de las cualidades del ensayo en modelo, en un amplio campo de casos, eliminando sus inconvenientes clásicos. El coste del estudio de una estructura muy compleja empleando Análisis Híbrido es inferior al coste mediante cálculo electrónico puro (software), mientras que el plazo puede ser del mismo orden.

TABLA 1. COMPARACION ENTRE LOS DISTINTOS METODOS

\begin{tabular}{l|c|c|c}
\hline \multicolumn{1}{c|}{ TABLA 1 } & $\begin{array}{c}\text { Ensayo } \\
\text { de } \\
\text { modelo }\end{array}$ & $\begin{array}{c}\text { Análisis } \\
\text { hibrido }\end{array}$ & $\begin{array}{c}\text { Cálculo } \\
\text { elec- } \\
\text { trónico }\end{array}$ \\
\hline Topología, material & + & + & - \\
Condiciones de borde & + & - & - \\
Hipótesis de carga & + & - & - \\
\hline
\end{tabular}

El signo + significa hardware; el - significa software.

El Análisis Híbrido reemplaza el punto más débil del cálculo numérico, que es la representación analítica del continuo elástico, por el modelo físico real (hardware). La introducción de condiciones de borde arbitrarias y sujetas a cambios, así como la generación de hipótesis de carga cualesquiera, se siguen haciendo numéricamente.

¿Cómo se consigue esta simbiosis entre el ordenador y el modelo reducido?

\section{LAS FUNCIONES DE GREEN}

El ordenador, para desempeñar el papel que tiene asignado en el Análisis Híbrido, debe recibir la información necesaria del modelo reducido en una forma perfectamente determinada.
Como es sabido, el comportamiento de un sólido elástico sometido a cualquier tipo de cargas y sustentado de cualquier forma en sus bordes, puede ser calculado con el solo conocimiento de las funciones de Green del sólido. Cada una de ellas da, para una determinada magnitud escalar (por ejempio, una de las componentes de la reacción o de la tensión en un punto del sólido), los valores que toma al actuar otra magnitud escalar unitaria (por ejemplo, una de las componentes de una fuerza o desplazamiento impuesto) en una serie de puntos del sólido elástico. Conocidas todas las funciones de Green, puede resolverse cualquier hipótesis de carga sin más que efectuar simples operaciones lineales (interpolación y superposición].

Las líneas y las superficies de influencia, tan útiles en el cálculo de puentes y de placas, no son sino casos particulares de funciones de Green.

Debe quedar claro que las funciones de Green representan un gigantesco repertorio de soluciones del problema elástico, de las cuales sólo una pequeña parte se utiliza posteriormente. Por ello, los programas complicados de cálculo electrónico (por ejemplo, por elementos finitos), cuya principal tarea es la descripción del sólido elástico, nunca se plantean la posibilidad de la obtención de todas estas soluciones superfluas. La estructura se calcula únicamente para un conjunto limitado de casos de cargas y de sustentaciones. Y por esa razón, no es posible tener en cuenta las modificaciones eventuales de las condiciones de apoyo.

Por el contrario, en el Análisis Híbrido, la parte de ensayo en modelo tiene como objetivo la medida directa de las funciones de Green que el ordenador necesita para realizar sus cálculos. Puesto que el modelo reducido reproduce naturalmente el comportamiento elástico de la estructura, la obtención de una gran cantidad de coeficientes de influencia puede automatizarse con mayor facilidad, por ejem plo, que la representación física de una hipótesis de carga.

Este método conduciría a la solución general del problema elástico si fuera posible en la 
práctica determinar exactamente las funciones de Green correspondientes a los desplazamientos producidos por la actuación de fuerzas. Otros efectos, como giros y deformaciones (o tensiones) y otras acciones, como momentos o deformaciones térmicas, podrían considerarse diferenciando esas funciones maestras. Pero, por una parte, no es posible que las fuerzas actúen sino en un número limitado (aunque grande) de puntos discretos, y por otra, los efectos están sujetos a un inevitable (aunque pequeño) error de medida. De todo ello resultan unas limitaciones prácticas al método, cuyo análisis pertenece al dominio del cálculo numérico.

\section{LA MATRIZ DE DATOS Y SU OBTENCION}

En el curso del desarrollo del Análisis Híbrido se ha llegado a la selección de un conjunto de funciones de Green adecuadas para su obtención en el ensayo y suficientes para el cálculo posterior en ordenador. Los datos su- ministrados por el modelo pueden agruparse en una matriz (fig. 1). Cada elemento de la matriz es un coeficiente de influencia; esto es, el efecto producido por una acción unitaria.

Las acciones consideradas son vectores fuerza o vectores desplazamiento impuesto, o sus componentes; y los efectos medidos son también fuerzas (reacciones de apoyo) y desplazamientos (flechas), así como deformaciones (o tensiones). Existiendo, pues, dos clases de acciones y tres clases de efectos, puede considerarse la matriz dividida en seis submatrices, cuyos elementos tienen dimensiones distintas. Como la relación de semejanza viene determinada por las dimensiones de cada magnitud, el ordenador puede transformar los valores medidos en el modelo en los valores correspondientes de la estructura real.

Para poder definir las funciones de influencia a partir de los valores de los coeficientes, el ordenador necesita conocer la situación de los puntos en que actúan las acciones y en que se miden los efectos, así como la direc-

\section{LA MATRIZ DE DATOS}

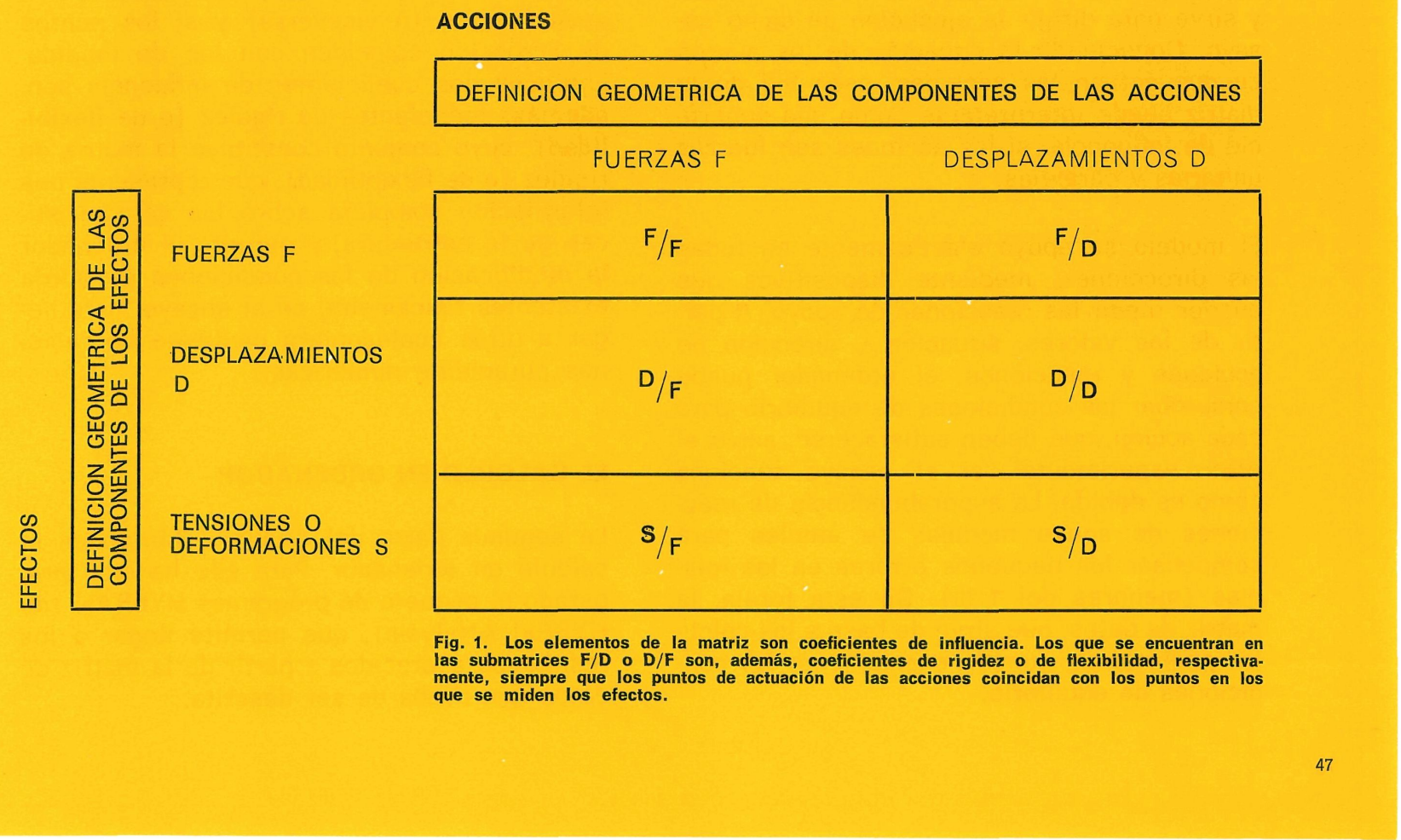




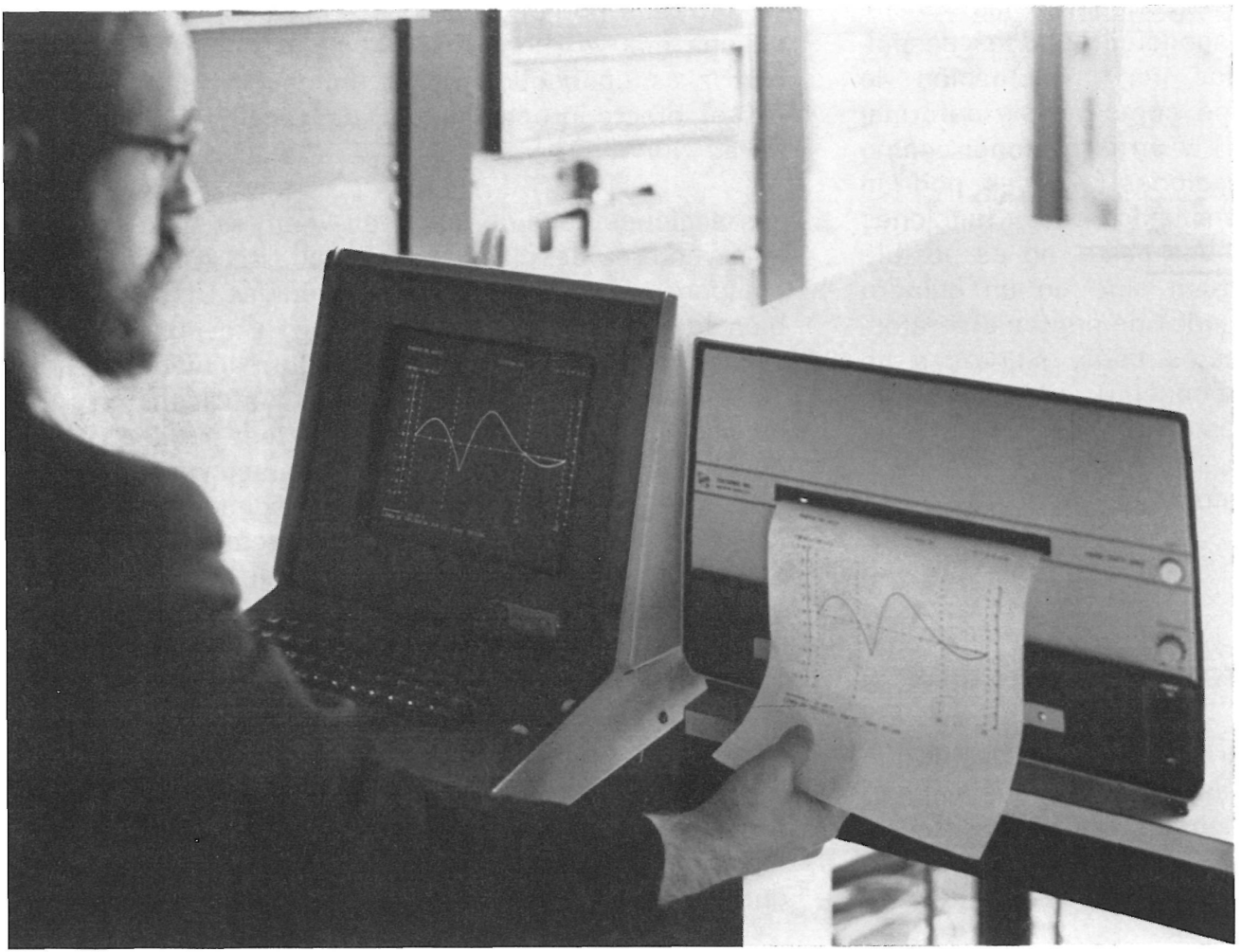

Fig. 2. Terminal de pantalla Tektronix, equipado ción de copias. La figura que aparece corres. ponde a una de las líneas de influencia que resultan del cálculo.

ción de cada uno de estos vectores. Esta información geométrica se introduce en el ordenador antes del ensayo, en forma tabular. y sirve para dirigir la ejecución de dicho ensayo. Conociendo la situación de los puntos en que actúan las acciones, cada fila de la matriz puede interpretarse como una superficie de influencia, si las acciones son fuerzas unitarias y paralelas.

El modelo se apoya elásticamente en todas las direcciones, mediante dispositivos que pueden medir las reacciones de apoyo. A partir de los valores, situación y dirección de acciones y reacciones, el ordenador puede comprobar las condiciones de equilibrio para cada acción, que deben satisfacerse, salvo el error experimental, si el ensayo funciona como es debido. La superabundancia de reacciones de apoyo medidas se emplea para compensar los pequeños errores en las mismas (menores del $1 \%$ ). De esta forma, la matriz de datos, que sirve de base a los cálculos posteriores, cumple exactamente las condiciones de equilibrio.
Los coeficientes de influencia adquieren un significado especial en el caso en que las acciones sean desplazamientos y los efectos sean fuerzas (o viceversa) y si los puntos de actuación coinciden con los de medida. Entonces, los coeficientes de influencia son, además, coeficientes de rigidez (o de flexibilidad), cuyo conjunto constituye la matriz de rigidez (o de flexibilidad), que representa una información completa sobre las características de la estructura, y permite al ordenador la modificación de las condiciones de borde existentes físicamente en el ensayo, para llegar a otras cualesquiera mediante operaciones puramente numéricas.

\section{EL CALCULO EN ORDENADOR}

La segunda parte del Análisis Híbrido es el cálculo en ordenador. Para ello ha sido preparado el paquete de programas HYBRAN (de HYBRid ANalysis), que permite llegar a los resultados apetecidos a partir de la matriz de datos que acaba de ser descrita. 
Este paquete de programas se caracteriza por una gran comodidad de utilización. Sus distintos módulos son completamente compatibles. Los resultados pueden obtenerse en forma numérica impresa o en forma gráfica en pantalla. En este último caso, un aparato reproductor suministra instantáneamente una copia de cada imagen de la pantalla. A continuación se pasa revista brevemente a las distintas partes del paquete.

Para empezar, la matriz de datos, procedente del modelo, se amplía para considerar un número arbitrario de efectos distintos de los medidos, mediante combinaciones lineales entre varias de sus filas. Así se obtienen giros diferenciando los desplazamientos; tensiones en cualquier dirección a partir de las deformaciones; etc.

Especialmente significativa es la obtención de funciones de influencia de esfuerzos en algunas secciones de la estructura.

Una vez obtenidos los coeficientes de influencia para todos los efectos que se juzgan de importancia para el análisis del comportamiento de la estructura, es preciso tener en cuenta las posibles diferencias entre la estructura real y la estructura ensayada, en cuanto a sus condiciones de borde. Como se ha dicho, en aquellos puntos en los que se conozca la rigidez, resulta posible modificar estas condiciones de borde. Así, un apoyo rígido puede transformarse en otro elástico o suprimirse por completo. En aquellos puntos en los que se tenga la matriz de rigidez entera (con sus seis libertades) puede conseguirse cualquier tipo de empotramiento espacial.

También resulta posible unir varias estructuras (que pueden haberse ensayado por separado), constituyendo así una estructura continua, siempre que sean conocidas las rigideces de los puntos de unión. A la inversa, pueden considerarse estructuras parciales, correspondientes a estados constructivos.

A partir de la matriz de datos correspondiente a la estructura (o estructuras) que se desea considerar, puede ya pasarse al cálculo de las distintas hipótesis de carga. Este cálculo, una vez conocidas las funciones de in- fluencia, se hace con una flexibilidad mucho mayor de la habitual en otros programas de cálculo electrónico, como se comprende fácilmente.

Además de las cargas usuales, que son las puntuales, lineales y superficiales, y de los desplazamientos (asientos) de apoyos, el método permite tratar con toda facilidad el caso de esfuerzos máximos para sobrecargas vivas, y el caso del pretensado considerado como carga.

En el caso de sobrecarga viva uniformemente repartida, no es preciso especificar su distribución. El ordenador, conociendo la superficie de influencia correspondiente, la distribuye de forma que se obtenga el esfuerzo más desfavorable, y comunica gráficamente al usuario la distribución elegida.

Parecido es el caso de trenes de carga que pueden moverse sobre la estructura a lo largo de vías prefijadas. El ordenador obtiene previamente las líneas de influencia que resultan de cortar las superficies de influencia a lo largo de las vías; y luego sitúa sobre estas líneas de influencia los trenes de carga en la posición más desfavorable.

En el caso del pretensado considerado como carga, también ofrece ventajas el empleo de las funciones de influencia, especialmente si hay que obtener, por tanteos, el trazado óptimo de los cables.

El programa ejecuta todo tipo de combinaciones entre los resultados de las distintas hipótesis de carga, como superposición simple, selección de valores máximos, superposición selectiva y multiplicación. Todas las matrices de datos y de resultados (intermedios y finales) son almacenadas en banda magnética por un período indefinido, de manera que estén siempre listas para su reutilización en nuevos cálculos. Esta medida revela su utilidad en muchos casos, como por ejemplo en las pruebas de carga de la estructura, que se efectúan meses o años después de su proyecto, permitiendo, en cuestión de minutos, la comparación de los valores procedentes de la estructura real con los que resultan del Análisis Híbrido. 


\section{LA TECNICA DEL ENSAYO Y DE LAS MEDIDAS}

La idea del Análisis Híbrido es fácilmente comprensible, simple e incluso trivial. Y no requiere medios considerables en la fase de cálculo, ya que, a pesar de tener un campo de aplicación mucho más grande que el del método de los Elementos Finitos, puede implementarse en un ordenador pequeño. El requisito previo del Análisis Híbrido, no obstante, es la aplicación de una técnica de ensayo y medidas muy por encima de la habitual en cuanto a precisión y velocidad; y esto ya no es tan trivial.

Es necesario que las funciones obtenidas experimentalmente sean diferenciables, y que las condiciones de apoyo puedan ser modificadas mediante manipulaciones de los datos medidos en el ensayo. Para ello, la mecánica de los dispositivos de ensayo, tanto respecto a los aparatos de apoyo como a los de aplicación de las cargas, debe satisfacer exactamente los requisitos teóricos. Los apoyos de- ben ser, por ejemplo, perfectamente rígidos o deformables sin ningún rozamiento, allí donde ello se requiera. Todos los elementos mecánicos deben sujetarse a normas de calidad muy estrictas, si se pretende que las operaciones numéricas posteriores conduzcan a los resultados correctos. Por ejemplo, si se desea obtener el coeficiente de rigidez de un apoyo con una precisión del $1 \%$, midiendo la reacción en dicho apoyo al aplicar un desplazamiento de 0,5 $\mathrm{mm}$, es necesario que el desplazamiento se aplique con un error menor de $0,005 \mathrm{~mm}$.

Lo mismo sucede con la técnica de medidas eléctricas. Sólo mediante procesos especialmente desarrollados al efecto es posible garantizar la corrección de todos y cada uno de los valores medidos, cuyo número alcanza con frecuencia las centenas de millar. Entre estos procesos merece mencionarse aquí el método de comparación 1 , que permite la compensación automática durante el ensayo de la influencia en el modelo de los factores variables en el tiempo (temperatura, humedad, características de los materiales, etc.).

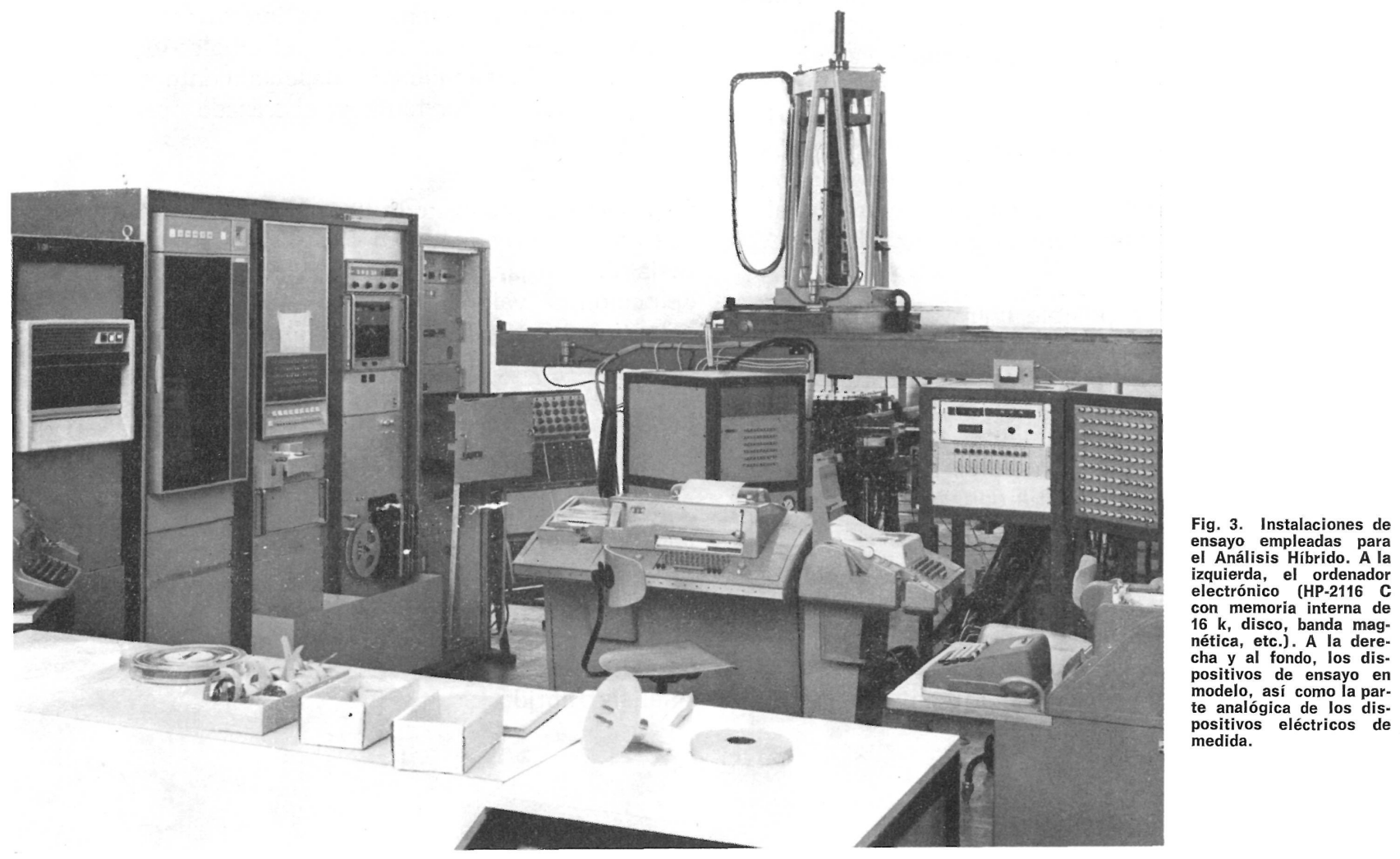


Fig. 4. Ejemplo número 5: Puente curvo $y$ en esviaje en Madrid. Proyectista: Prof. J. A. Torroja.
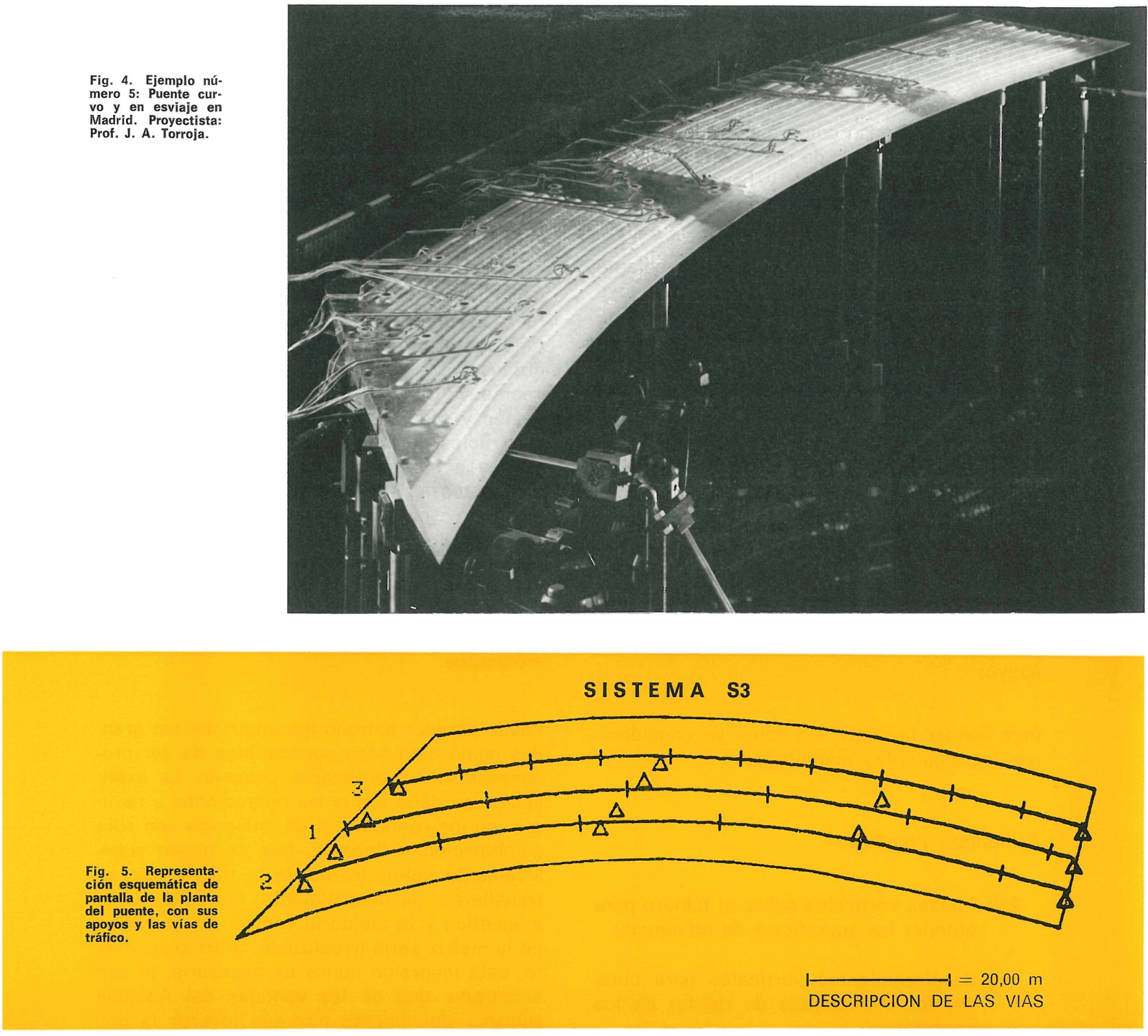

La automatización completa del ensayo es imprescindible no sólo por la velocidad nece. saria, sino para permitir la reproducción exacta de un ensayo independientemente de influjos humanos. En unión con el ordenador se abre así la posibilidad de mejorar significativamente la precisión de las medidas, aplicando a las mismas los métodos de la Estadística.

El conjunto de instalaciones de ensayo para Análisis Híbrido desarrollado en el laboratorio del autor, siguiendo los criterios que se han mencionado, puede verse en la figura 3 .

\section{EJEMPLOS DE APLICACIONES PRACTICAS}

Los ejemplos de aplicación del Análisis Híbrido que se mencionan a continuación son una muestra de la actividad del laboratorio en los últimos meses. De los cuatro primeros se ofrece nada más una descripción muy somera en las figuras correspondientes, mientras que el quinto se estudia con más detalle.

Se trata de un puente losa pretensado, de planta curva y apoyos en esviaje (fig. 4). La losa es continua sobre tres vanos, y su sección va aligerada mediante la introducción de 
tubos que funcionan como encofrado perdido. El puente debe servir de paso superior sobre una autopista en Madrid, y ha sido proyectado por el Prof. J. A. Torroja.

Una de las cuestiones a resolver mediante el ensayo era si los cuatro puntos de apoyo previstos en la segunda fila de soportes (figura 5) eran realmente necesarios, o si, por el contrario, podían suprimirse los soportes internos o los externos. También debía estudiarse la influencia de la elasticidad de los apoyos en el reparto de las reacciones verticales entre los distintos soportes del puente.

Se preparó un modelo del puente en plexiglás, a escala $1: 50$, y respetando cuidadosamente todos los detalles geométricos del mismo. El modelo se colocó sobre 13 soportes biarticulados y se sujetó frente a movimientos horizontales mediante un sistema isostático de apoyos.

Para formar la matriz de datos se consideraron las siguientes magnitudes:

Acciones actuantes:

230 fuerzas verticales sobre el tablero para obtener las superficies de influencia;

13 desplazamientos verticales para obtener los coeficientes de rigidez de los soportes;

Efectos medidos:

186 deformaciones (62 rosetas) en las caras superior e inferior;

13 reacciones en los apoyos;

4 flechas en la zona más sensible de la estructura (parte inferior del vano izquierdo) .

Para cada una de las acciones consideradas se medieron todos los efectos citados. Es decir, la matriz de datos constaba de $243 \times 201=$
$=48.843$ elementos (coeficientes de influencia). Una vez obtenidos, el ensayo se dio por terminado, desmontándose el modelo para dejar plaza para el ensayo siguiente.

Comenzó luego el cálculo numérico en ordenador. Ante todo, se amplió la matriz de datos. Las deformaciones se transformaron en tensiones referidas al sistema general de coordenadas que se empleaba para describir la geometría del modelo, así como la de las acciones y efectos. También se obtuvieron las superficies de influencia de las tensiones referidas a sistemas locales de coordenadas, dispuestos paralelamente al eje curvo del puente. Se obtuvieron, por último, las superficies de influencia de los tres esfuerzos a tener en cuenta (esfuerzo cortante y momentos flector y torsor) en 15 secciones del puente. Con todo ello, la matriz de datos a emplear en los cálculos posteriores alcanzó los 108.621 elementos.

Puede parecer extraño que matrices tan grandes como ésta sean susceptibles de ser procesadas en un ordenador pequeño. La explicación se debe a que las operaciones a realizar con los coeficientes de influencia son sólo combinaciones lineales, que se hacen sucesivamente sobre trozos de la matriz que se transfieren secuencialmente entre el disco magnético y la memoria central. La inversión de la matriz sería irrealizable; afortunadamente, esta inversión nunca es necesaria, lo que constituye una de las ventajas del Análisis Híbrido. Unicamente hay que invertir la matriz de rigidez o la matriz de flexibilidad cuando se desea cambiar las condiciones de apoyo, y estas matrices, en el ejemplo considerado. tienen sólo $13 \times 13=169$ elementos.

La estructura considerada en la matriz de datos descrita (puente apoyado en 13 soportes) se llamó estructura S2. A partir de la matriz se obtuvo otra de iguales dimensiones, correspondiente a la nueva estructura S3, constituida por el mismo puente una vez suprimidos los dos soportes exteriores de la segunda fila. Las hipótesis de carga se prepararon teniendo en cuenta la Instrucción Española. Se consideraron primero las cargas permanentes, separando el peso propio y la carga muerta. Fácilmente se comprende que los datos de 
cada hipótesis de carga son válidos independientemente de cuál sea el sistema de apoyos considerado, por lo cual basta con introducirlos una sola vez en el ordenador para que éste calcule los resultados correspondientes.

A continuación se obtuvieron los esfuerzos máximos para la hipótesis de sobrecarga uniformemente repartida en la posición más desfavorable. En la figura 6 pueden verse algunos resultados correspondientes a esta hipótesis. El tren de cargas a considerar consiste en un carro de $60 \mathrm{Mp}$, que puede moverse sobre una cualquiera de las tres vías que se definen en la figura 5. Para calcular sus posiciones más desfavorables se prepararon las líneas de influencia, a lo largo de las tres vías, de todos los efectos considerados en las dos estructuras. Resultan, por tanto, $2 \times 3 \times 447=2.682$ líneas de influencia, al gunas de las cuales pueden verse en las figuras 9 a 16, reproducidas directamente de la pantalla del ordenador. Puede observarse la continuidad de estas curvas, que han sido obtenidas por interpolación a partir de valores aislados de coeficientes de influencia procedentes del ensayo.

Los resultados obtenidos demuestran claramente que el criterio para decidir la supresión de los dos soportes exteriores está determinado por el valor de la tensión en las proximidades del soporte inferior situado a la derecha del vano principal. Por ello, se han representado en las figuras mencionadas las lineas de influencia de esta tensión.

También se han representado (figs. 7 y 8) las tensiones en la cara superior del puente en la hipótesis de cargas permanentes.

Por último, es preciso elegir el pretensado longitudinal $\mathrm{y}$, sobre todo, el transversal sobre la fila de soportes considerada, de modo que se reduzcan convenientemente las tracciones peligrosas. Para ello se calculó previamente el efecto de distintos pretensados unitarios. A la vista de estos resultados, el pro- yectista pudo encontrar un pretensado óptimo, que le permitió finalmene tomar la decisión de suprimir los dos soportes exteriores. Para terminar, se recalculó el puente con el pretensado elegido y se obtuvieron los esfuerzos máximos para las distintas combinaciones entre las hipótesis de carga.

De esta forma queda expuesto el desarrollo completo del cálculo de un puente mediante el Análisis Híbrido, junto con los resultados intermedios y su representación gráfica.

\section{HYBRAN COMO PROGRAMA DE CALCULO PURAMENTE NUMERICO}

El sistema de programas HYBRAN fue desarrollado para satisfacer los requisitos del Análisis Híbrido. Ningún otro programa de cálculo sigue el procedimiento de obtener las funciones de influencia antes de iniciar el cálculo de una hipótesis de carga determinada.

No obstante, la experiencia de su uso ha demostrado que ese procedimiento también constituye una herramienta de gran flexibilidad en manos del proyectista de una estructura de barras calculable numéricamente mediante programas tradicionales, y que puede competir con dichos programas.

Para posibilitar esta forma de cálculo se ha preparado un programa general para estructuras de barras, que genera las funciones de Green (en este caso líneas de influencia) en la forma precisa en que deben ser recibidas por el programa HYBRAN.

El paquete de programas resultante, al que se ha añadido también un programa de cálculo de secciones, está perfectamente listo para su utilización práctica. No es éste, sin embargo, lugar para entrar en detalles acerca de sus cualidades, como son una compatibilidad completa; la salida de resultados gráficos en pantalla (ver fig. 2); la selección de resultados fácilmente interpretables, etc., etc. 


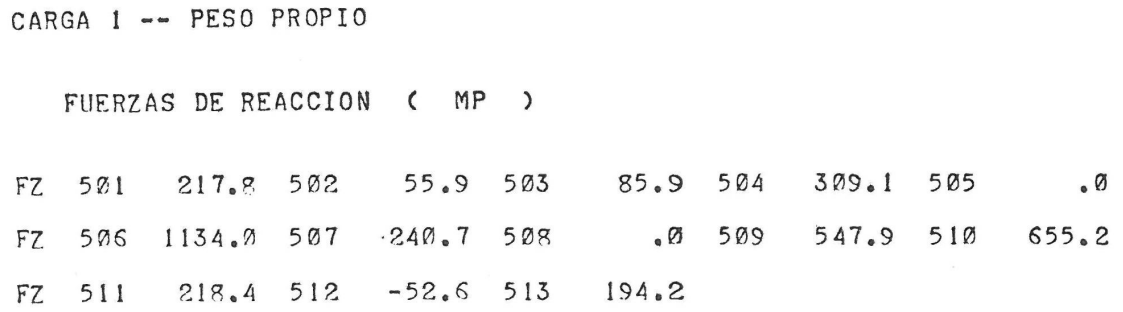

CARGA 1 - PESO PROPIO

FIIERZAS DE REACCION (MP )

\begin{tabular}{|c|c|c|c|c|c|c|c|c|c|c|c|}
\hline & $F Z$ & 501 & 213.7 & 592 & 43.3 & 503 & 60.4 & 504 & 340.7 & 505 & 867.4 \\
\hline \multirow{2}{*}{$\begin{array}{l}\text { Fig. 6. Reacciones en los } \\
13 \text { apoyos en las estructuras } \\
\text { S2 y S3. La numeración de } \\
\text { los apoyos va de izquierda } \\
\text { a derecha, según la figura } 5 \text {. }\end{array}$} & $\mathrm{FZ}$ & 506 & 73.7 & 507 & -30.6 & 508 & 488.0 & 569 & 572.6 & 510 & 617.1 \\
\hline & $F Z$ & 511 & 222.2 & 512 & -49.4 & 513 & 187.5 & & & & \\
\hline
\end{tabular}

CARGA 1 - PESO PROPIO

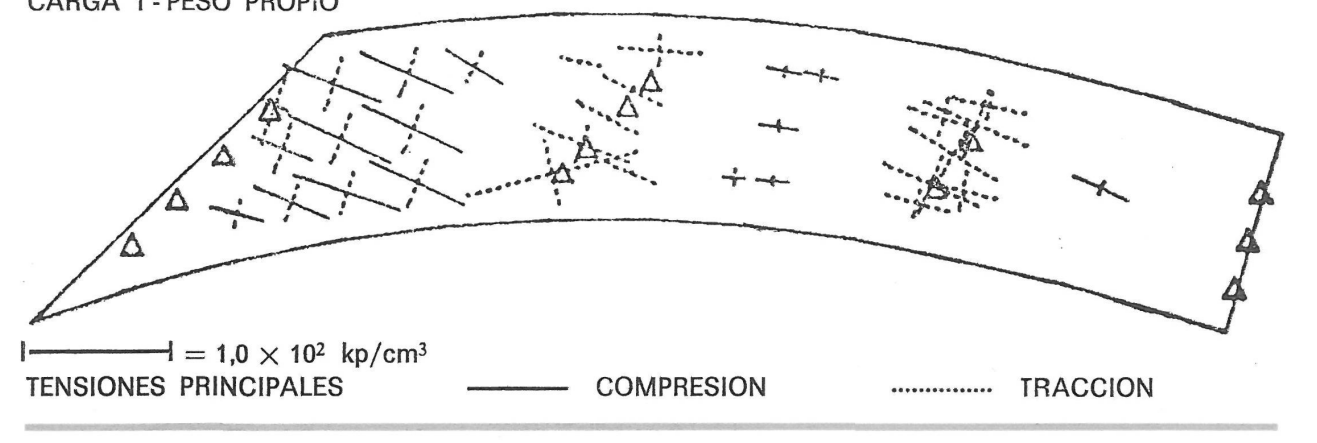

Figs. 7 y 8 . Tensiones principales en la cara superior del taturas S2 y S3. En el caso de la estructura S3 han desaparecido los dos apoyos exteriores de la segunda fila; como puede verse en la figura 6, su nula.

CARGA 1 - PESO PROPIO

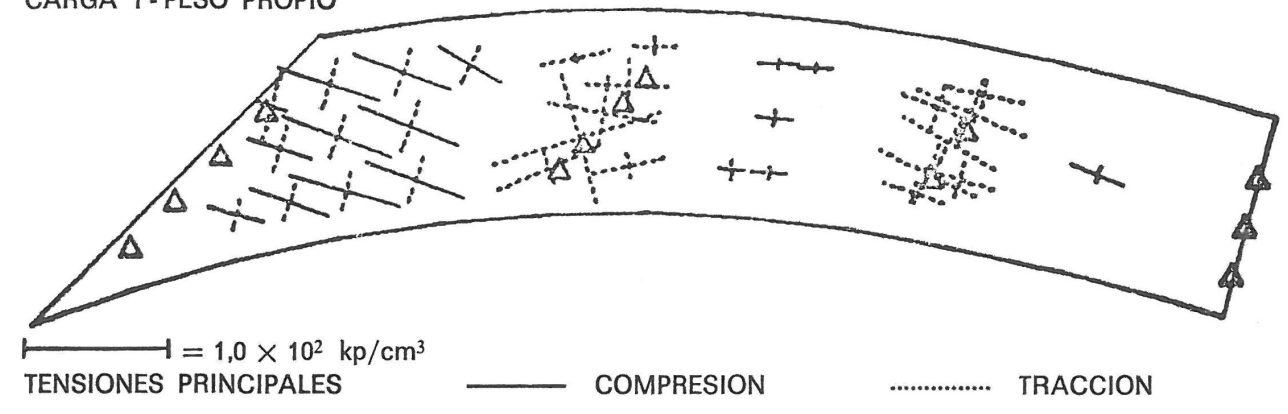




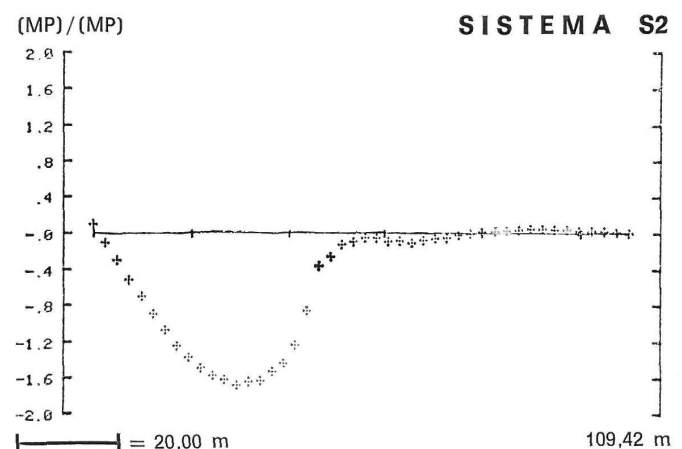

LINEA DE INFLUENCIA A LO LARGO DE VIA 2 PARA FZ 505 (MP) / (MP)

SISTEMA \$2

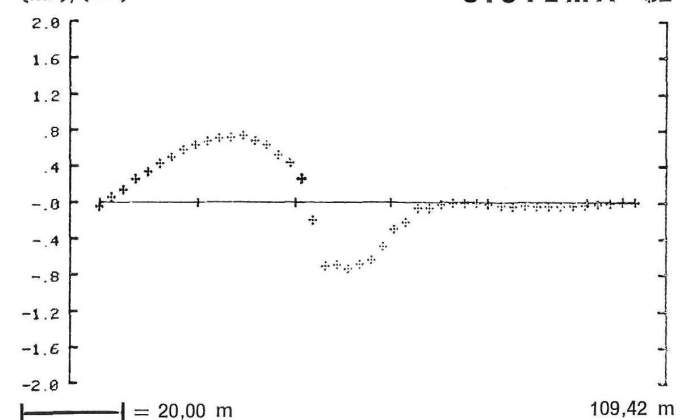

LINEA DE INFLUENCIA A LO LARGO DE VIA 2 PARA FZ 506 (MP) / (MP)

SISTEMA S3

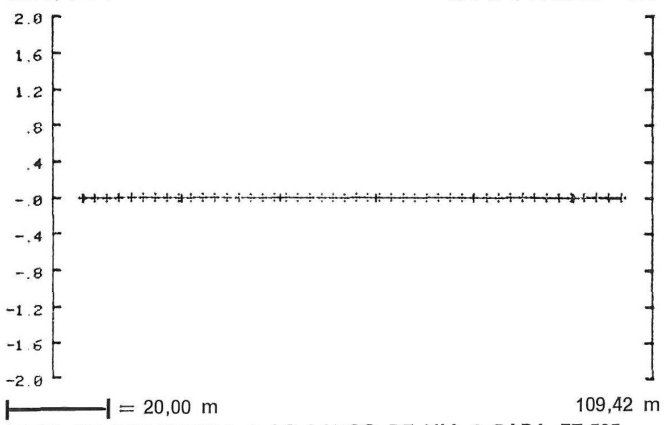

LINEA DE INFLUENCIA A LO LARGO DE VIA 2 PARA FZ 505 (MP) / (MP)

SISTEMA S3

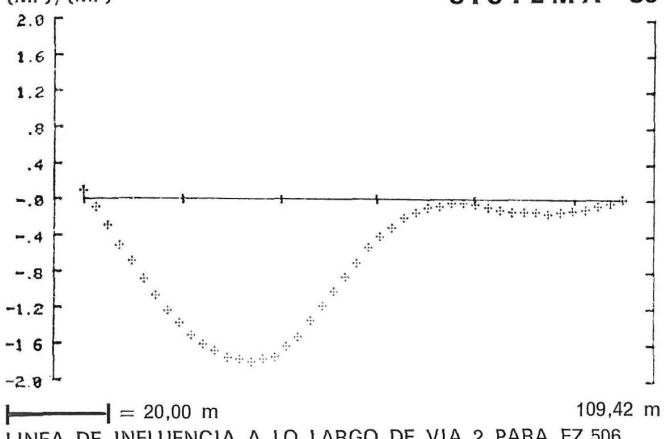

LINEA DE INFLUENCIA A LO LARGO DE VIA 2 PARA FZ 506

Figs. 9 a 12. Líneas de influencia de las reacciones en dos apoyos de la segunda fila de las estructuras S2 y S3 para una carga que recorre la vía número 2. El apoyo 505 ha sido suprimido en la estructura S3, y la linea de influencia correspondiente al apoyo 506 ha sido obtenida numericamente a partir de las $\left(\mathrm{kp} / \mathrm{cm}^{2}\right) /(\mathrm{MP}) \times 10^{-1}$

SISTEMA S2

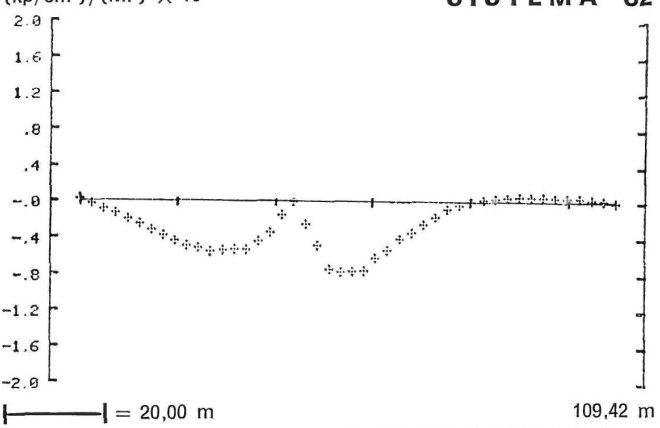

LINEA DE INFLUENCIA A LO LARGO DE VIA 2 PARA SU 827 $\left(\mathrm{kp} / \mathrm{cm}^{2}\right) /(\mathrm{MP}) \times 10^{-1}$

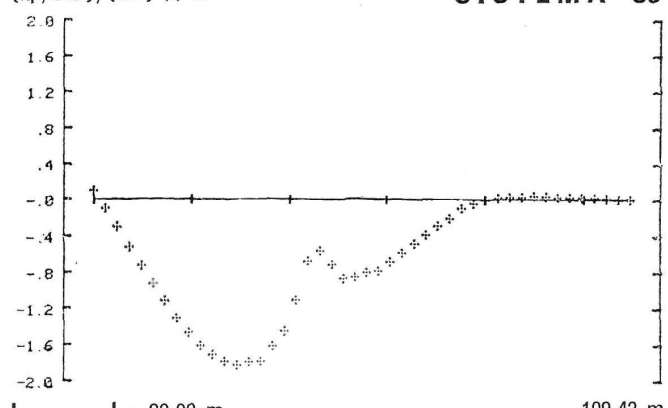

LINEA DE INFLUENCIA A LO LARGO DE VIA 2 PARA SU 827

$\left(\mathrm{kp} / \mathrm{cm}^{2}\right) /(\mathrm{MP}) \times 10^{-1}$

SISTEMA S3

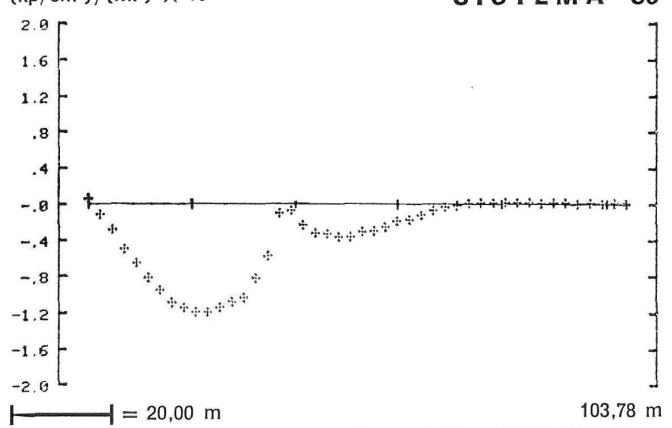

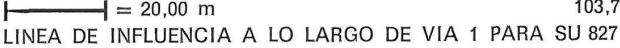

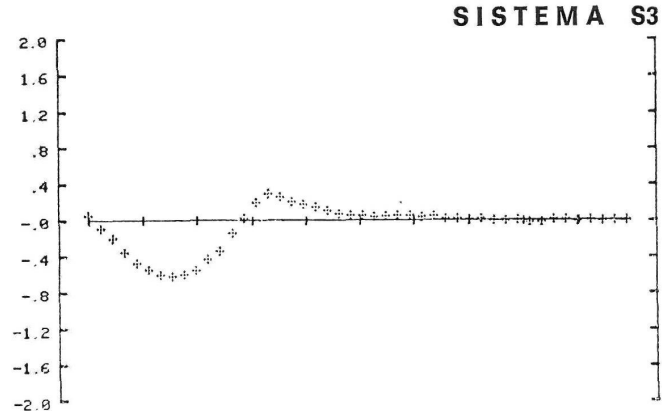

L-2.9 $=20,00 \mathrm{~m} \quad 98,71 \mathrm{~m}$ LINEA DE INFLUENCIA A LO LARGO DE VIA 3 PARA SU 827

Figs. 13 a 16 . Líneas de influencia de la componente de la tensión SU sobre el soporte 506, en la dirección del eje del puente, para carga que recorre la vía número 2 en ambas estructuras. 


\section{ejemplos}

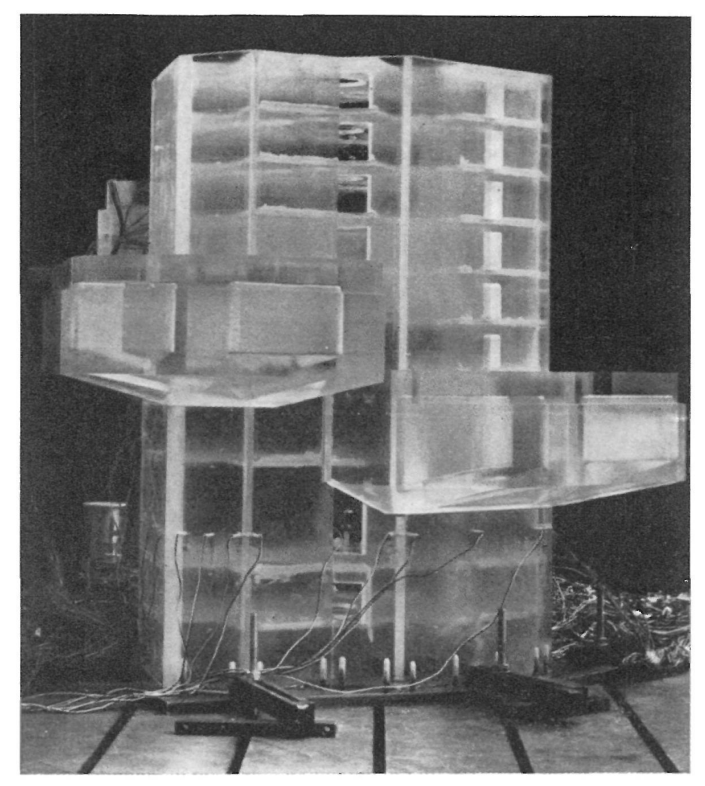

Fig. 17. Encargo: Investigación del comportamiento resistente de las mensulas en cajón de forma especial, que soportan en su borde los pilares de fachada del edificio de 40 pisos, y de la transmisión de los esfuerzos de estos elementos al núcleo del edificio, de forma poligonal con algunos aligeramientos.
Objeto: Nuevo edificio para el National Westminster Bank, en Londres.

Estudio encargado por: Pell, Frǐschmann and Partners, Ingenieros consultores, de Londres.

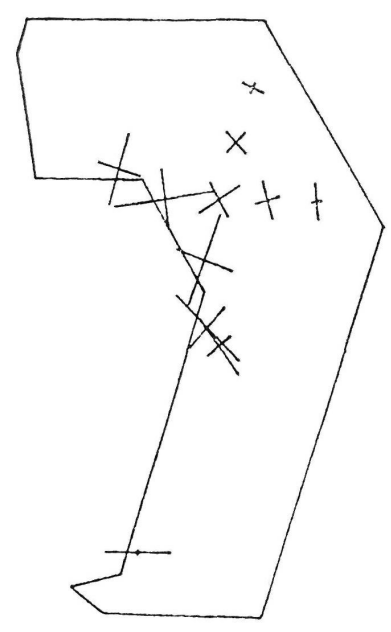

$=10,00 \mathrm{kp} / \mathrm{cm}^{2}$

TENSIONES PRINCIPALES

COMPRESION

TRACCION

Fig. 18. Distribución de las tensiones principales en la cara superior de la ménsula bajo carga máxima en e borde.

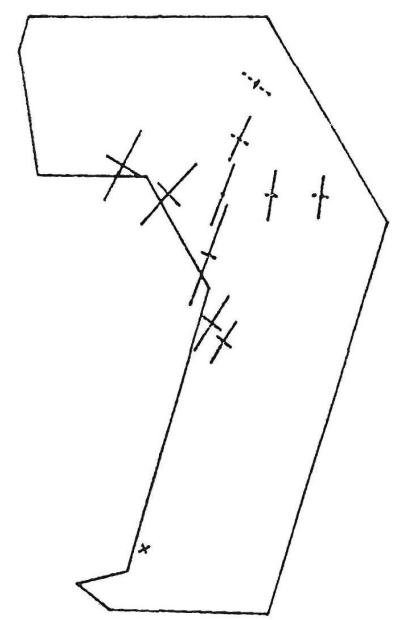

$\longmapsto=10,00 \mathrm{kp} / \mathrm{cm}^{2}$

TENSIONES PRINCIPALES

COMPRESION

TRACCION

Fig. 19. Distribución de las tensiones principales en la cara superior de la ménsula bajo una carga aislada en 


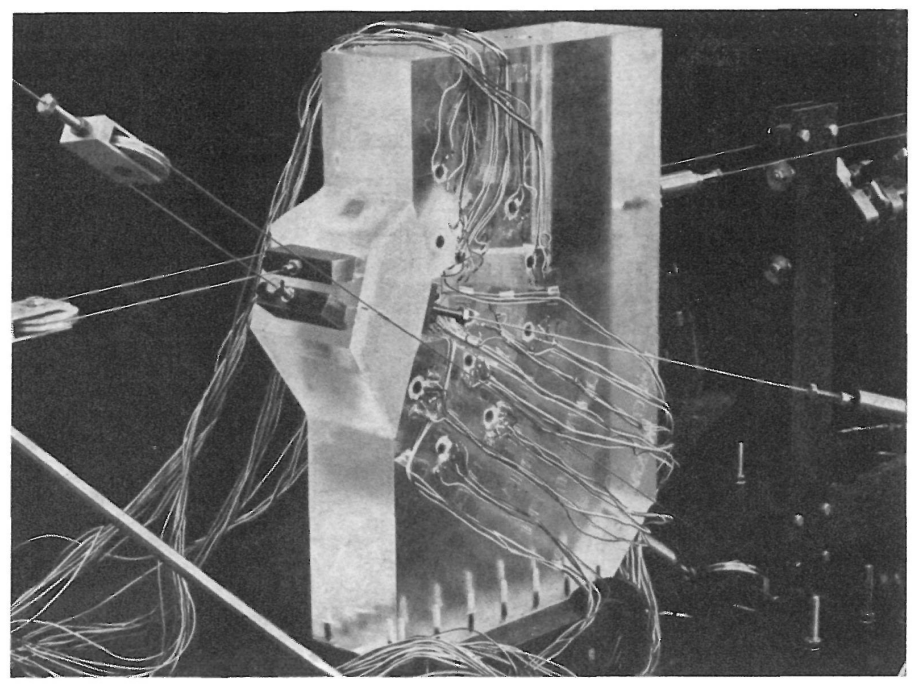

Fig. 20. Encargo: Investigación de la distribución de tensiones del contra fuerte, en la zona de transmisión de los esfuerzos de la compuerta giratoria, bajo varias hipotesis de carga simetricas y antimétricas, asi como

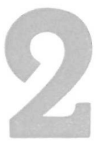

Objeto: Contrafuerte de Valeira, en Portugal. Estudio encargado por: Stahlton AG, Zurich.

SISTEMA SO

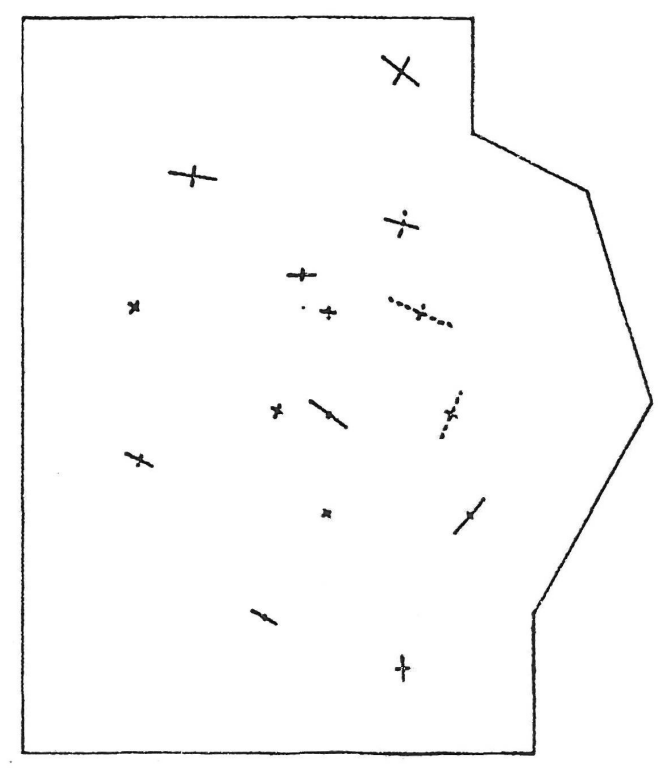

$-1=50,00 \mathrm{kp} / \mathrm{cm}^{2}$

TENSIONES PRINCIPALES COMPRESION TRACCION

Fig. 22. Distribución de las tensiones bajo carga simétrica de la compuerta.
SISTEMA SO

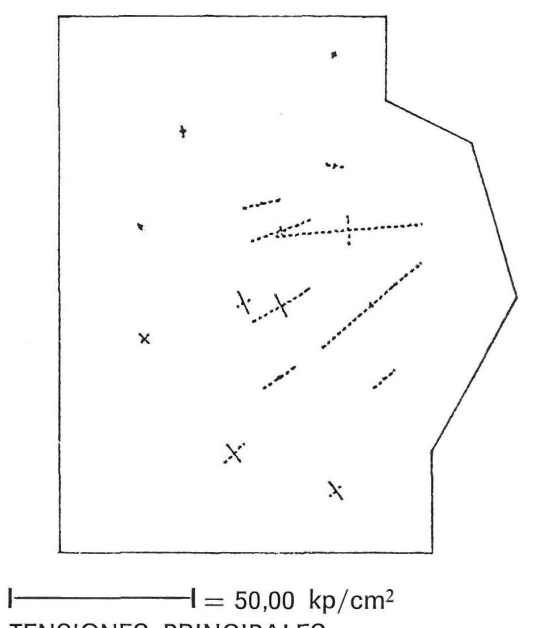

TENSIONES PRINCIPALES COMPRESION

TRACCION

Fig. 21. Distribución de las tensiones bajo pretensado
SISTEMA SO

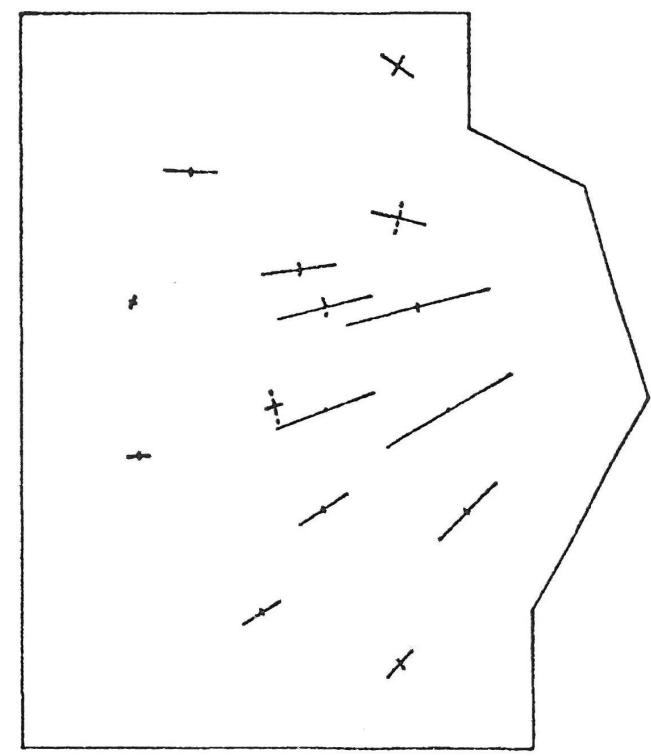

$-1=50,00 \mathrm{kp} / \mathrm{cm}^{2}$

TENSIONES PRINCIPALES

COMPRESION

TRACCION

Fig. 23. Combinación de pretensado y carga de la compuerta. 
Objeto: Edificio M.G.B., en Zurich.

Estudio encargado por: Altorfer, Cogliatti y Schellenberg, Ingenieros, de Zurich.

LASTFALL 203 - GRENZWERTE AUS WIND UND ERDBEBEN

SELEKTION DER EXTREMWERTE AUS:

201 -- GRENZWERTE AUS DEN WINDBELASTUNGEN

$202=$ GRENZWERTE AUS DEN ERDBEBENBELASTUNGEN

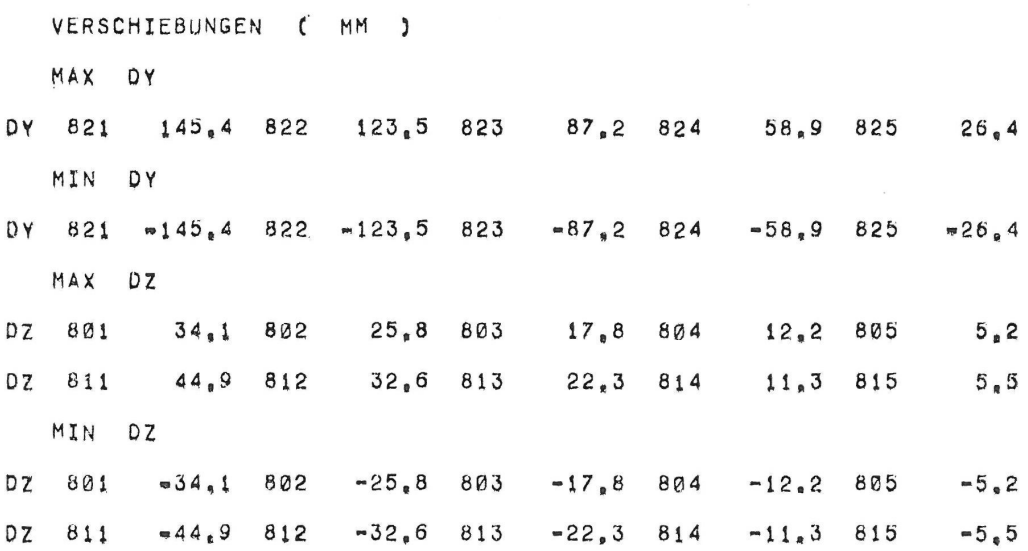

26.4

26.4

5.2

5,5

$-5.2$

$-5.5$

\section{SPANNUNGEN (KP/CH2)}

$$
\text { MAX } S X
$$

SX $101 \quad 3,0102$

$\begin{array}{llllll}5 \times \quad 107 & 6.3 & 108 & 10.1 & 109\end{array}$
$.8 \quad 104$

$2,6 \quad 206$

6.8291

8.5202

7.1207

8,2208

$5.6 \quad 343$

15.330

$26.9 \quad 309$

15.1401

$23.8 \quad 407$

22,1503

$47.7 \quad 502$

28.4548

33.2539

$42.9 \quad 507$

1.9
3.2
21.0
15.9
28.7
10.5
45.9
45.1

Fig. 25. Tanto para la carga de viento como para la de sismo se consideraron 8 direcciones. El cálculo se simplificó extraordinariamente partiendo de las funciones de intorsores. Los esfuerzos máximos se obtuvieron automáticamente (ver listado de ordenador).

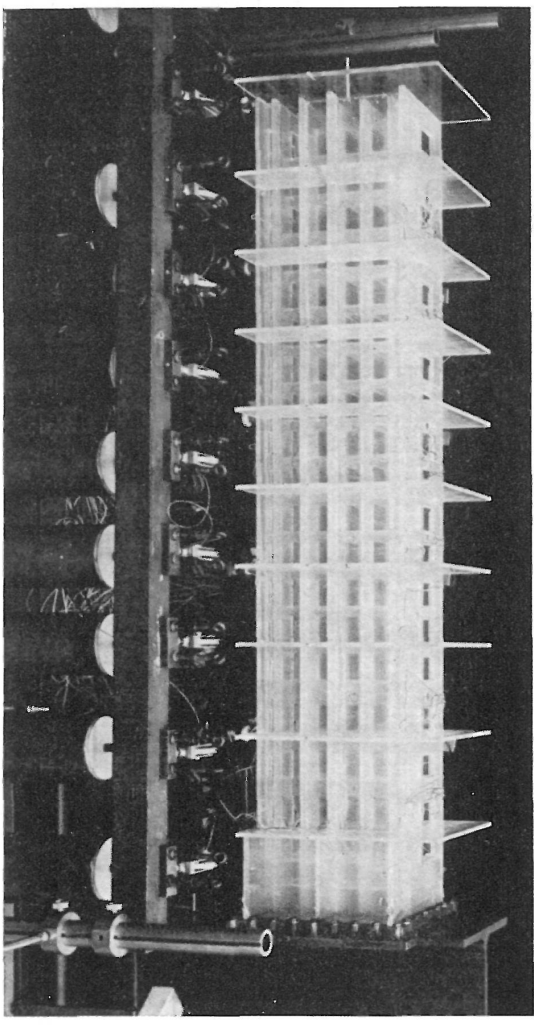

Fig. 24. Encargo: Investigación del comportamiento resistente del núcleo de ascensores bajo carga de
viento y sismo con diferentes direcciones y varias excentricidades. 


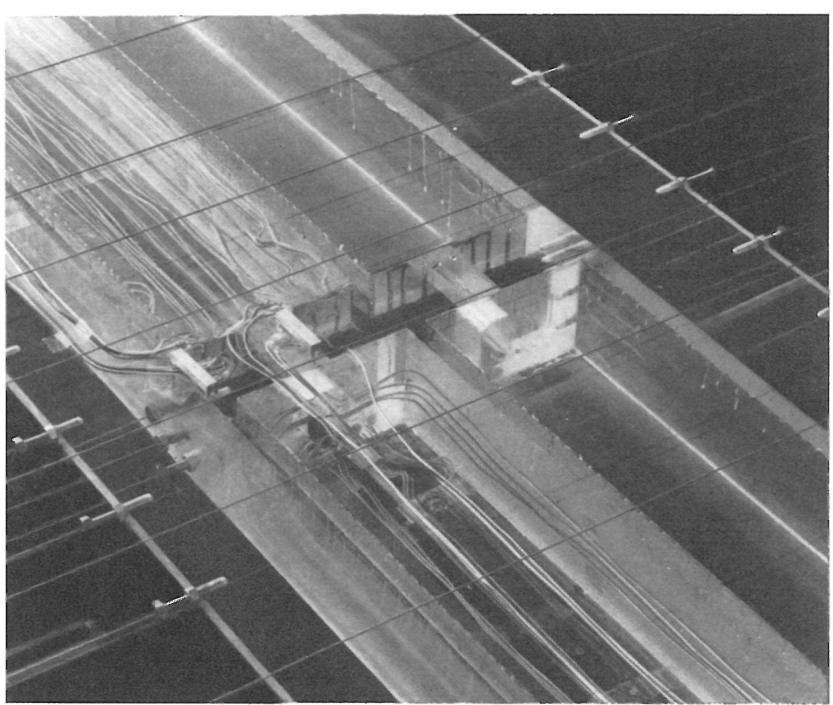

Fig. 26. Encargo: Investigación del cargadero sobre la pila doble. Estudio especial de la distribución de tensiones debidas al pretensado del cargadero, as cho eficaz) y de la distribución de tensiones alrededor de las aberturas situa. das sobre los apoyos.
Objeto: Viaducto sobre el valle de Vinxbach.

Estudio encargado por: Polensky y Zöllner, Empresa Constructora, de Francfort del Main.

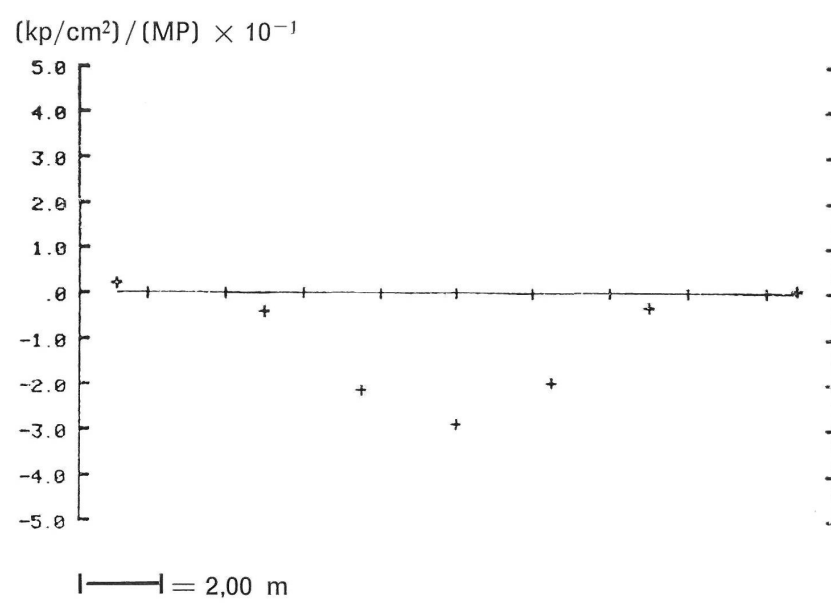

LINEA DE INFLUENCIA DEBIDA A FZ PARA: SY 381

Fig. 27. Línea de influencia de la tensión transversal en el cargadero debida a un pretensado unitario del tablero. Con ello queda determinado el ancho eficaz para este caso de carga.

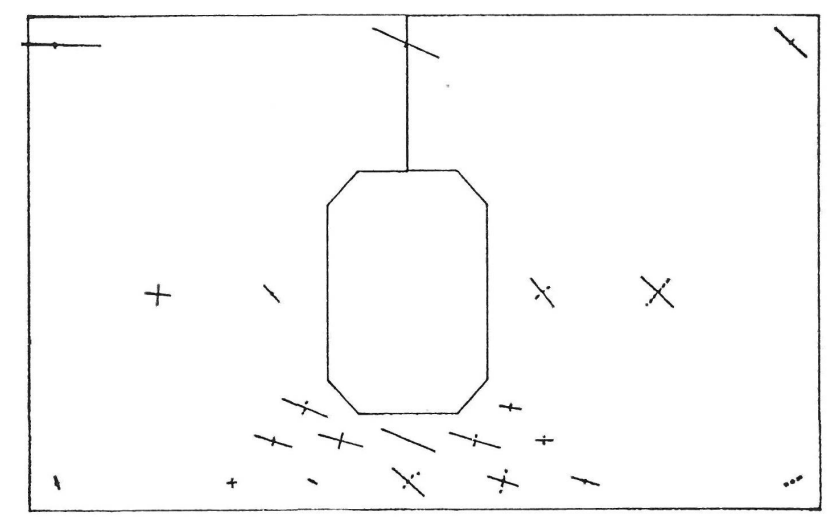

$\mathrm{I}=50,00 \mathrm{kp} / \mathrm{cm}^{2}$

COMPRESION

TRACCION

TENSIONES PRINCIPALES

Fig. 28. Tensiones principales en el cargadero debidas a pretensado transversal total.

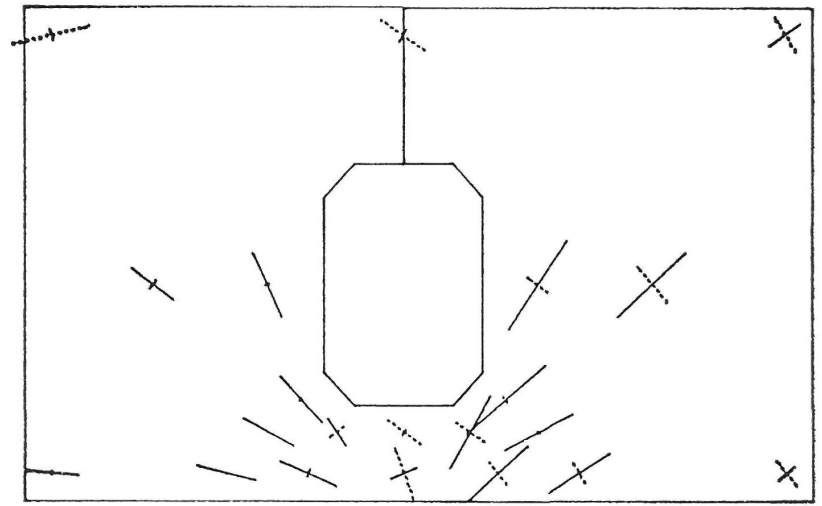

$\mathrm{I} \longrightarrow=5,00 \mathrm{kp} / \mathrm{cm}^{2}$

COMPRESION

TRACCION

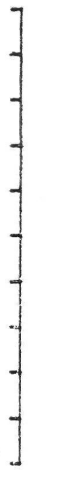




\section{résumé}

\section{Analyse hybride}

Heinz Hossdorf, ingénieur civil

Au cours des dernières années on a développé, parallèlement à la méthode des éléments finites, une autre méthode particulièrement conçue pour le calcul de structures élastiques complexes: l'analyse hybride. Cette nouvelle et puissante méthode est encore peu connue, même chez les spécialistes, bien qu'elle soit, depuis peu, appliquée avec succès dans la pratique.

L'analyse hybride est, en bon nombre de cas, préférable aux méthodes numériques pures, tant pour des raisons économiques que pour des raisons techniques, parmi lesquelles ressort son domaine d'application plus ample.

Bien que son exposition soit conceptuellement simple, la méthode exige une technologie très spécialisée et un système de programmes de calcul électronique qui ne pourraient pas être décrits en détail dans un article aussi court que celui-ci. Par conséquent, l'auteur expose ici seulement les idées servant de base à la méthode, aussi bien que ses possibilités, et traite de son application à quelques cas concrets.

Pour un traitement plus détaillé de l'analyse hybride et des techniques d'essai correspondantes, on peut consulter le livre intitulé «Modelos reducidos («Modèles réduits»), H. Hossdorf, édite par I'Institut Eduardo Torroja, Madrid, 1972.

\section{summary}

Hybrid analysis

Heinz Hossdorf, Civil Engineer

Recent years have seen the development, along with the Finite Elements Method, of another which is particularly conceived for the calculation of complex elastic structures: the Hybrid Analysis. This new and powerful method is still relatively unkown even among specialists, although it has been applied for some time now with successful results.

In many cases, the Hybrid Analysis is preferable to pure numerical methods, both for economic and technical reasons among which is that fact that its range of application is much wider.

Althoug conceptually simple, the method requires a very specialized technology and a system of electronic computation programs that cannot be described in detail in an article as short as this. For this reason, only the basic ideas of this method will be discussed below while its possibilities will be illustrated by showing its application in specific cases.

For a more detailed discussion of the Hybrid Analysis and of its corresponding test techniques, see the book "Modelos reducidos», by H. Hossdorf, published by the Instituto Eduardo Torroja, Madrid, 1972.

\section{zusammenfassung}

\section{Hybridenanalyse}

Heinz Hossdorf, Ingenieur

In den letzten Jahren hat sich parallel zur Methode der begrenzten Elemente eine andere. Methode entwickelt, die besonders zur Kalkulation von elastischen, komplexen Strukturen dient. Diese neue, potentielle Methode ist bis jetzt kaum bekannt, sogar unter den Spezialisten, obwohl sie seit einiger Zeit erfolgreich in der Praxis benutzt wird.

Die Hybridenanalyse ist den reinen, numerischen Methoden, die sie durch ihren weiten Anwendungsbereich und aus finanziellen Grúnden übertrifft, in vielen Fällen vorzuziehen.

Obwohl die Darstellung ler Analyse im Konzept einfach ist, erfordert das System doch eine sehr spezielle Technik und eine elektronische Rechnungsprogrammierung, die in einem so kurzen Artikel wie diesem nicht ausführlich beschrieben werden kann. Deshalb werden anschliessend nur die Grundideen der Methode angeführt und die Anwendungsmöglichkeiten durch Angabe von Beispielen erläutert.

Eine detaillierte Behandlung der Hybridenanalyse und der entsprechenden Versuchstechniken bietet das Buch "Reduzierte Modelle", von H. Hossdorf, das 1972 vom Institut Eduardo Torroja herausgegeben wurde. 